\title{
On some properties of split Horadam quaternions
}

\author{
Dorota Bród \\ Rzeszow University of Technology, \\ Faculty of Mathematics and Applied Physics, \\ al. Powstańców Warszawy 12, \\ 35-959 Rzeszów, Poland \\ email: dorotab@prz.edu.pl
}

\begin{abstract}
In this paper we introduce and study the split Horadam quaternions. We give some identities, among others Binet's formula, Catalan's, Cassini's and d'Ocagne's identities for these numbers.
\end{abstract}

\section{Introduction}

Let $\mathbb{C}$ be the field of complex numbers. A quaternion $x$ is a hyper-complex number represented by

$$
\mathbb{H}=\left\{x=a_{0}+a_{1} \mathbf{i}+a_{2} \mathbf{j}+a_{3} \mathbf{k}: a_{s} \in \mathbb{R}, s=0,1,2,3\right\},
$$

where $\{\mathbf{1}, \mathbf{i}, \mathbf{j}, \mathbf{k}\}$ is an orthonormal basis in $\mathbb{R}^{4}$, which satisfies the quaternion multiplication rules:

$$
\begin{gathered}
\mathbf{i}^{2}=\mathbf{j}^{2}=\mathbf{k}^{2}=\mathbf{i j k}=-1, \\
\mathbf{i j}=\mathbf{k}=-\mathbf{j i}, \mathbf{j k}=\mathbf{i}=-\mathbf{k j}, \mathbf{k i}=\mathbf{j}=-\mathbf{i k} .
\end{gathered}
$$

The quaternions were introduced by W. R. Hamilton in 1843 .

2010 Mathematics Subject Classification: 11B37, 11R52

Key words and phrases: Horadam numbers, quaternion, split quaternion, split Horadam quaternion 
Another extension of the complex numbers is the algebra of split quaternions. The split quaternions were introduced by J. Cockle in 1849 [2]. The set of split (or coquaternions) can be represented as

$$
\widehat{\mathbb{H}}=\left\{y=b_{0}+b_{1} i+b_{2} j+b_{3} k: b_{s} \in \mathbb{R}, s=0,1,2,3\right\},
$$

where $\{1, i, j, k\}$ is the basis of $\mathbb{H}$ satisfying the following equalities

$$
\begin{gathered}
\mathfrak{i}^{2}=-j^{2}=-k^{2}=-1, \\
i j=k=-j i, j k=-i=-k j, k i=j=-i k .
\end{gathered}
$$

The split quaternion can be rewritten as

$$
y=\left(b_{0}+b_{1} i\right)+\left(b_{2}+b_{3} i\right) j=z_{1}+z_{2} j, z_{1}, z_{2} \in \mathbb{C} .
$$

The split quaternions contain nontrivial zero divisors, nilpotent elements and idempotents. The conjugate of a split quaternion $y=b_{0}+b_{1} i+b_{2} j+b_{3} k$, denoted by $\bar{y}$, is given by $\bar{y}=b_{0}-b_{1} i-b_{2} j-b_{3} k$. The norm of $y$ is defined as

$$
\mathrm{N}(\mathrm{y})=\mathrm{y} \overline{\mathrm{y}}=\mathrm{b}_{0}^{2}+\mathrm{b}_{1}^{2}-\mathrm{b}_{2}^{2}-\mathrm{b}_{3}^{2} .
$$

Let $y_{1}, y_{2} \in \hat{\mathbb{H}}, y_{1}=a_{1}+b_{1} i+c_{1} j+d_{1} k, y_{2}=a_{2}+b_{2} i+c_{2} j+d_{2} k$. Then addition and subtraction of the split quaternions is defined as follows

$$
y_{1} \pm y_{2}=\left(a_{1} \pm a_{2}\right)+\left(b_{1} \pm b_{2}\right) i+\left(c_{1} \pm c_{2}\right) j+\left(d_{1} \pm d_{2}\right) k .
$$

Multiplication of the split quaternions is defined by

$$
\begin{aligned}
y_{1} \cdot y_{2}= & a_{1} a_{2}-b_{1} b_{2}+c_{1} c_{2}+d_{1} d_{2}+\left(a_{1} b_{2}+b_{1} a_{2}-c_{1} d_{2}+d_{1} c_{2}\right) i \\
& +\left(a_{1} c_{2}+c_{1} a_{2}-b_{1} d_{2}+d_{1} b_{2}\right) j+\left(a_{1} d_{2}+d_{1} a_{2}+b_{1} c_{2}-c_{1} b_{2}\right) k .
\end{aligned}
$$

For the basics on split quaternions theory, see [5].

\section{The Horadam numbers}

In [3] Horadam introduced a sequence $\left\{W_{n}\right\}$ defined by the following relation

$$
W_{0}=a, W_{1}=b, W_{n}=p W_{n-1}+q W_{n-2} \text { for } n \geq 2
$$

for arbitrary $a, b, p, q \in \mathbb{Z}$. This sequence is a certain generalization of famous sequences such as Fibonacci sequence $\left\{F_{n}\right\}(a=0, b=1, p=q=1)$, Lucas 
sequence $\left\{L_{n}\right\}(a=2, b=1, p=q=1)$, Jacobsthal sequence $\left\{J_{n}\right\}(a=0, b=$ $1, p=1, q=2)$, Pell sequence $\left\{P_{n}\right\}(a=0, b=1, p=2, q=1)$, Pell-Lucas sequence $\left\{P L_{n}\right\}(a=b=1, p=2, q=1)$. The sequences defined by (5) are called sequences of the Fibonacci type.

The characteristic equation associated with the recurrence (5) is

$$
r^{2}-p r-q=0
$$

Assuming that $p^{2}+4 q>0$, the equation has the following roots

$$
r_{1}=\frac{p+\sqrt{p^{2}+4 q}}{2}, r_{2}=\frac{p-\sqrt{p^{2}+4 q}}{2} .
$$

Note that

$$
\begin{aligned}
r_{1}+r_{2} & =p \\
r_{1}-r_{2} & =\sqrt{p^{2}+4 q}, \\
r_{1} r_{2} & =-q .
\end{aligned}
$$

The Binet's formula for the sequence $\left\{W_{n}\right\}$ has the following form

$$
W_{n}=\frac{\left(b-a r_{2}\right) r_{1}^{n}-\left(b-a r_{1}\right) r_{2}^{n}}{r_{1}-r_{2}}
$$

Let

$$
\alpha=\frac{b-a r_{2}}{r_{1}-r_{2}}, \beta=\frac{b-a r_{1}}{r_{1}-r_{2}}
$$

Then

$$
W_{n}=\alpha r_{1}^{n}-\beta r_{2}^{n}
$$

In the next section we will use the following result.

Theorem 1 Let $\mathrm{n}, \mathrm{p}, \mathrm{q}$ be integers such that $\mathrm{n} \geq 0, \mathrm{p}^{2}+4 \mathrm{q}>0$. Then

$$
\sum_{l=0}^{n-1} W_{l}=\frac{W_{n}+q W_{n-1}+a(p-1)-b}{p+q-1} .
$$

Proof. Using formula (11), (7) and (9), we get

$$
\sum_{l=0}^{n-1} W_{l}=\sum_{l=0}^{n-1}\left(\alpha r_{1}^{l}-\beta r_{2}^{l}\right)=\alpha \frac{1-r_{1}^{n}}{1-r_{1}}-\beta \frac{1-r_{2}^{n}}{1-r_{2}}
$$




$$
\begin{aligned}
& =\frac{\alpha-\beta-\left(\alpha r_{2}-\beta r_{1}\right)-\left(\alpha r_{1}^{n}-\beta r_{2}^{n}\right)+r_{1} r_{2}\left(\alpha r_{1}^{n-1}-\beta r_{2}^{n-1}\right)}{1-\left(r_{1}+r_{2}\right)+r_{1} r_{2}} \\
& =\frac{\alpha-\beta-\left(\alpha r_{2}-\beta r_{1}\right)-W_{n}-q W_{n-1}}{1-p-q} .
\end{aligned}
$$

By simple calculations we have $\alpha-\beta=a, \alpha r_{2}-\beta r_{1}=a p-b$. Hence

$$
\sum_{l=0}^{n-1} W_{l}=\frac{W_{n}+q W_{n-1}+a(p-1)-b}{p+q-1} .
$$

Numbers of the Fibonacci type appear in many subjects of mathematics. In [4] Horadam defined the Fibonacci and Lucas quaternions. In [1] the split Fibonacci quaternions $Q_{n}$ and split Lucas quaternions $T_{n}$ were introduced by the following relations

$$
\begin{aligned}
Q_{n} & =F_{n}+i F_{n+1}+j F_{n+2}+k F_{n+3}, \\
T_{n} & =L_{n}+i L_{n+1}+j L_{n+2}+k L_{n+3},
\end{aligned}
$$

where $F_{n}, L_{n}$ is $n$th Fibonacci and Lucas number, resp. and $\{i, j, k\}$ is the standard basis of split quaternions. In the literature there are many generalizations of the Fibonacci and Lucas sequences, among others k-Fibonacci sequence $\left\{F_{k, n}\right\}$, k-Lucas sequence $\left\{L_{k, n}\right\}$, defined for $k \in \mathbb{N}$ in the following way

$$
\begin{aligned}
& \mathrm{F}_{k, 0}=0, \mathrm{~F}_{k, 1}=1, \mathrm{~F}_{k, n}=\mathrm{kF}_{k, n-1}+\mathrm{F}_{k, n-2} \text { for } \mathrm{n} \geq 2, \\
& \mathrm{~L}_{k, 0}=2, \mathrm{~L}_{k, 1}=k, \mathrm{~L}_{k, n}=k \mathrm{~L}_{k, n-1}+\mathrm{L}_{k, n-2} \text { for } \mathrm{n} \geq 2 .
\end{aligned}
$$

Some interesting results for the split k-Fibonacci and split k-Lucas quaternions can be found in [6]. In [7] the authors studied split Pell quaternions $S_{n}$ and split Pell-Lucas quaternions $S P L_{n}$ defined by

$$
\begin{aligned}
S P_{n} & =P_{n}+i P_{n+1}+j P_{n+2}+k P_{n+3}, \\
S P L_{n} & =P L_{n}+i P L_{n+1}+j P L_{n+2}+k P L_{n+3},
\end{aligned}
$$

where $P_{n}$ and $P L_{n}$ is $n$th Pell and Pell-Lucas number, resp.

We will focus on split Horadam quaternions. We will present some identities for the split Horadam quaternions, which generalize the results for the split Fibonacci quaternions, the split Lucas quaternions, the split Pell quaternions and the split Pell-Lucas quaternions. 


\section{The split Horadam quaternions}

For $n \geq 0$ define the split Horadam quaternion $\mathrm{H}_{\mathrm{n}}$ by

$$
H_{n}=W_{n}+i W_{n+1}+j W_{n+2}+k W_{n+3} \text {, }
$$

where $W_{n}$ is the $n$th Horadam number and $i, j, k$ are split quaternionic units which satisfy the multiplication rules given by (1) and (2).

By (5) and (13) we obtain

$$
\begin{aligned}
\mathrm{H}_{0}= & a+b i+j(p b+q a)+k\left(p^{2} b+p q a+q b\right) \\
H_{1}= & b+i(p b+q a)+j\left(p^{2} b+p q a+q b\right)+k\left(p^{3} b+p^{2} q a+2 p q b+q^{2} a\right) \\
H_{2}= & p b+q a+i\left(p^{2} b+p q a+q b\right)+j\left(p^{3} b+p^{2} q a+2 p q b+q^{2} a\right) \\
& +k\left(p^{4} b+p^{3} q a+2 p q(p b+q a)+p^{2} q b+q^{2} b\right)
\end{aligned}
$$

For any $n \geq 0$ we obtain the norm of $H_{n}$.

Proposition 1 Let $\mathrm{n}, \mathrm{p}, \mathrm{q}$ be integers such that $\mathrm{n} \geq 0, \mathrm{p}^{2}+4 \mathrm{q}>0$. Then

$$
\begin{aligned}
N\left(H_{n}\right)= & \left(1-q^{2}-p^{2} q^{2}\right) W_{n}^{2}+\left(1-p^{2}-\left(p^{2}+q^{2}\right)^{2}\right) W_{n+1}^{2} \\
& -2 p q\left(1+p^{2}+q\right) W_{n} W_{n+1} .
\end{aligned}
$$

Proof. Using formula (3) and (13), we get

$$
\begin{aligned}
N\left(H_{n}\right)= & W_{n}^{2}+W_{n+1}^{2}-W_{n+2}^{2}-W_{n+3}^{2} \\
= & \left.W_{n}^{2}+W_{n+1}^{2}-\left(p W_{n+1}+q W_{n}\right)^{2}-\left(\left(p^{2}+q\right) W_{n+1}+p q W_{n}\right)\right)^{2} \\
= & W_{n}^{2}+W_{n+1}^{2}-\left(p^{2} W_{n+1}^{2}+2 p q W_{n} W_{n+1}+q^{2} W_{n}^{2}\right) \\
& -\left(\left(p^{2}+q\right)^{2} W_{n+1}^{2}+2 p q\left(p^{2}+q\right) W_{n} W_{n+1}+p^{2} q^{2} W_{n}^{2}\right) .
\end{aligned}
$$

By simple calculations we get the result.

By (13) we get a recurrence relation for the split Horadam quaternions.

Proposition 2 Let $\mathrm{n}, \mathrm{p}, \mathrm{q}$ be integers such that $\mathrm{n} \geq 2, \mathrm{p}^{2}+4 \mathrm{q}>0$. Then

$$
\mathrm{H}_{\mathrm{n}}=\mathrm{pH}_{\mathrm{n}-1}+\mathrm{qH}_{\mathrm{n}-2} \text {, }
$$

where $\mathrm{H}_{0}, \mathrm{H}_{1}$ are given by (14). 
Proof. By formula (13) and (5) we get

$$
\begin{aligned}
\mathrm{pH}_{n-1}+q H_{n-2}= & p\left(W_{n-1}+i W_{n}+j W_{n+1}+k W_{n+2}\right) \\
& +q\left(W_{n-2}+i W_{n-1}+j W_{n}+k W_{n+1}\right) \\
= & p W_{n-1}+q W_{n-2}+i\left(p W_{n}+q W_{n-1}\right) \\
& +j\left(p W_{n+1}+q W_{n}\right)+k\left(p W_{n+2}+q W_{n+1}\right) \\
= & W_{n}+i W_{n+1}+j W_{n+2}+k W_{n+3}=H_{n},
\end{aligned}
$$

which ends the proof.

Theorem 2 Let $\mathrm{n}, \mathrm{p}, \mathrm{q}$ be integers such that $\mathrm{n} \geq 0, \mathrm{p}^{2}+4 \mathrm{q}>0$. Then

(i) $\mathrm{H}_{\mathrm{n}}+\overline{\mathrm{H}_{\mathrm{n}}}=2 \mathrm{~W}_{\mathrm{n}}$,

(ii) $\mathrm{N}\left(\mathrm{H}_{\mathrm{n}}\right)=2 \mathrm{~W}_{\mathrm{n}} \mathrm{H}_{\mathrm{n}}-\mathrm{H}_{\mathrm{n}}^{2}$.

Proof. (i) Using the definition of the conjugate of a split quaternion we obtain the result.

(ii) By formula (13) we have

$$
\begin{aligned}
H_{n}^{2}= & W_{n}^{2}-W_{n+1}^{2}+W_{n+2}^{2}+W_{n+3}^{2} \\
& +2 i W_{n} W_{n+1}+2 j W_{n} W_{n+2}+2 k W_{n} W_{n+3} \\
= & -W_{n}^{2}-W_{n+1}^{2}+W_{n+2}^{2}+W_{n+3}^{2} \\
& +2\left(W_{n}^{2}+i W_{n} W_{n+1}+j W_{n} W_{n+2}+k W_{n} W_{n+3}\right) \\
= & 2 W_{n}\left(W_{n}+i W_{n+1}+j W_{n+2}+k W_{n+3}\right) \\
& -W_{n}^{2}-W_{n+1}^{2}+W_{n+2}^{2}+W_{n+3}^{2} \\
= & 2 W_{n} H_{n}-N\left(H_{n}\right) .
\end{aligned}
$$

Hence we get the result.

The next theorem presents the Binet's formula for the split Horadam quaternions.

Theorem 3 (Binet's formula) Let $\mathrm{n}, \mathrm{p}, \mathrm{q}$ be integers such that $\mathrm{n} \geq 0, \mathrm{p}^{2}+$ $4 q>0$. Then

$$
H_{n}=\alpha \hat{r}_{1} r_{1}^{n}-\beta \hat{r}_{2} r_{2}^{n}
$$

where $r_{1}, r_{2}, \alpha, \beta$ are given by (6), (10), resp. and $\hat{r}_{1}=1+i r_{1}+j r_{1}^{2}+k r_{1}^{3}$, $\widehat{r_{2}}=1+i r_{2}+j r_{2}^{2}+k r_{2}^{3}$. 
Proof. By (11) we have

$$
\begin{aligned}
\mathrm{H}_{n}= & W_{n}+i W_{n+1}+j W_{n+2}+k W_{n+3} \\
= & \alpha r_{1}^{n}-\beta r_{2}^{n}+i\left(\alpha r_{1}^{n+1}-\beta r_{2}^{n+1}\right)+j\left(\alpha r_{1}^{n+2}-\beta r_{2}^{n+2}\right) \\
& +k\left(\alpha r_{1}^{n+3}-\beta r^{n+3}\right) \\
= & \alpha r_{1}^{n}\left(1+i r_{1}+j r_{1}^{2}+k r_{1}^{3}\right)-\beta r_{2}^{n}\left(1+i r_{2}+j r_{2}^{2}+k r_{2}^{3}\right) \\
= & \alpha \hat{r}_{1} r_{1}^{n}-\beta \hat{r}_{2} r_{2}^{n} .
\end{aligned}
$$

Using the Binet's formula (15), we can obtain some new identities for the split Horadam quaternions. We will use the following lemma.

Lemma 1 Let $\hat{r_{1}}=1+i r_{1}+j r_{1}^{2}+k r_{1}^{3}, \hat{r_{2}}=1+i r_{2}+j r_{2}^{2}+k r_{2}^{3}$, where $r_{1}, r_{2}$ are given by (6). Then

$$
\begin{aligned}
\hat{r_{1}} \hat{r_{2}}= & 1+q+q^{2}-q^{3}+i\left(p+q^{2} \sqrt{p^{2}+4 q}\right) \\
& +j\left(p^{2}+2 q-p q \sqrt{p^{2}+4 q}\right)+k\left(p^{3}+3 p q+q \sqrt{p^{2}+4 q}\right), \\
\hat{r_{2} \hat{r_{1}}=} & 1+q+q^{2}-q^{3}+i\left(p-q^{2} \sqrt{p^{2}+4 q}\right) \\
& +j\left(p^{2}+2 q+p q \sqrt{p^{2}+4 q}\right)+k\left(p^{3}+3 p q-q \sqrt{p^{2}+4 q}\right) .
\end{aligned}
$$

Proof. Using formula (4), we have

$$
\begin{aligned}
\hat{r_{1}} \hat{r_{2}}= & 1-r_{1} r_{2}+\left(r_{1} r_{2}\right)^{2}+\left(r_{1} r_{2}\right)^{3}+i\left(r_{1}+r_{2}+\left(r_{1} r_{2}\right)^{2}\left(r_{1}-r_{2}\right)\right) \\
& +j\left(r_{1}^{2}+r_{2}^{2}+r_{1} r_{2}\left(r_{1}^{2}-r_{2}^{2}\right)\right)+k\left(r_{1}^{3}+r_{2}^{3}-r_{1} r_{2}\left(r_{1}-r_{2}\right)\right), \\
\widehat{r_{2}} \hat{r}_{1}= & 1-r_{1} r_{2}+\left(r_{1} r_{2}\right)^{2}+\left(r_{1} r_{2}\right)^{3}+i\left(r_{1}+r_{2}-\left(r_{1} r_{2}\right)^{2}\left(r_{1}-r_{2}\right)\right) \\
& +j\left(r_{1}^{2}+r_{2}^{2}-r_{1} r_{2}\left(r_{1}^{2}-r_{2}^{2}\right)\right)+k\left(r_{1}^{3}+r_{2}^{3}+r_{1} r_{2}\left(r_{1}-r_{2}\right)\right) .
\end{aligned}
$$

By (7) and (9) we get

$$
\begin{aligned}
& r_{1}^{2}+r_{2}^{2}=\left(r_{1}+r_{2}\right)^{2}-2 r_{1} r_{2}=p^{2}+2 q \\
& r_{1}^{3}+r_{2}^{3}=\left(r_{1}+r_{2}\right)^{3}-3 r_{1} r_{2}\left(r_{1}+r_{2}\right)=p^{3}+3 p q .
\end{aligned}
$$

Hence

$$
\begin{aligned}
\hat{r_{1}} \hat{r_{2}}= & 1+q+q^{2}-q^{3}+i\left(p+q^{2} \sqrt{p^{2}+4 q}\right) \\
& +j\left(p^{2}+2 q-p q \sqrt{p^{2}+4 q}\right)+k\left(p^{3}+3 p q+q \sqrt{p^{2}+4 q}\right),
\end{aligned}
$$




$$
\begin{aligned}
\hat{r_{2}} \hat{r_{1}}= & 1+q+q^{2}-q^{3}+i\left(p-q^{2} \sqrt{p^{2}+4 q}\right) \\
& +j\left(p^{2}+2 q+p q \sqrt{p^{2}+4 q}\right)+k\left(p^{3}+3 p q-q \sqrt{p^{2}+4 q}\right) .
\end{aligned}
$$

\section{Corollary 1}

$$
\hat{r_{1}} \hat{r_{2}}+\hat{r_{2}} \hat{r_{1}}=2\left(1+q+q^{2}-q^{3}+p i+j\left(p^{2}+2 q\right)+k\left(p^{3}+3 p q\right)\right) .
$$

Theorem 4 (Catalan's identity) Let $\mathrm{n}, \mathrm{m}, \mathrm{p}, \mathrm{q}$ be integers such that $\mathrm{n} \geq \mathrm{m}$, $p^{2}+4 q>0$. Then

$$
H_{n-m} H_{n+m}-H_{n}^{2}=\alpha \beta(-q)^{n-m}\left[(-q)^{m}\left(\hat{r_{1}} \hat{r_{2}}+\hat{r_{2}} \hat{r_{1}}\right)-r_{2}^{2 m} \hat{r_{1}} \hat{r_{2}}-r_{1}^{2 m} \hat{r_{2}} \hat{r_{1}}\right] \text {, }
$$

where $\alpha, \beta, \widehat{r_{1}} \hat{r_{2}}+\widehat{r_{2}} \hat{r_{1}}, \hat{r_{1}} \hat{r_{2}}, \hat{r_{2}} \widehat{r_{1}}$ are given by (10), (18), (16), (17), resp.

Proof. By (15) we get

$$
\begin{aligned}
H_{n-m} H_{n+m}-H_{n}^{2}= & \left(\alpha \hat{r_{1}} r_{1}^{n-m}-\beta \hat{r_{2}} r_{2}^{n-m}\right)\left(\alpha \hat{r_{1}} r_{1}^{n+m}-\beta \hat{r_{2}} r_{2}^{n+m}\right) \\
& -\left(\alpha \hat{r_{1}} r_{1}^{n}-\beta \hat{r_{2}} r_{2}^{n}\right)\left(\alpha \hat{r_{1}} r_{1}^{n}-\beta \hat{r_{2}} r_{2}^{n}\right) \\
= & \alpha \beta\left(r_{1} r_{2}\right)^{n-m}\left[\left(r_{1} r_{2}\right)^{m}\left(\hat{r_{1}} \hat{r_{2}}+\hat{r_{2}} \hat{r_{1}}\right)\right. \\
& \left.-r_{2}^{2 m} \hat{r_{1}} \hat{r_{2}}-r_{1}^{2 m} \hat{r_{2}} \hat{r_{1}}\right]
\end{aligned}
$$

Using formula (9), we obtain

$$
H_{n-m} H_{n+m}-H_{n}^{2}=\alpha \beta(-q)^{n-m}\left((-q)^{m}\left(\hat{r_{1}} \widehat{r_{2}}+\widehat{r_{2}} \hat{r_{1}}\right)-r_{2}^{2 m} \hat{r_{1}} \widehat{r_{2}}-r_{1}^{2 m} \widehat{r_{2}} \hat{r_{1}}\right) \text {. }
$$

Corollary 2 (Cassini's identity) Let $\mathrm{n}, \mathrm{p}, \mathrm{q}$ be integers such that $\mathrm{n} \geq 0, \mathrm{p}^{2}+$ $4 q>0$. Then

$$
H_{n-1} H_{n+1}-H_{n}^{2}=-\alpha \beta(-q)^{n-1}\left(q\left(\hat{r_{1}} \hat{r_{2}}+\hat{r_{2}} \hat{r_{1}}\right)+r_{2}^{2} \hat{r_{1}} \widehat{r_{2}}+r_{1}^{2} \hat{r_{2}} \hat{r_{1}}\right) .
$$

Note that for $\mathrm{p}=\mathrm{q}=1$ we get the Cassini's identity for the split Fibonacci quaternions $Q_{n}$ and the split Lucas quaternions $T_{n}([1])$.

Corollary 3 Let $\mathrm{n} \geq 1$ be an integer. Then

(i) $\mathrm{Q}_{n-1} \mathrm{Q}_{\mathrm{n}+1}-\mathrm{Q}_{\mathrm{n}}^{2}=(-1)^{\mathrm{n}}\left(2 \mathrm{Q}_{1}-2 \mathrm{i}-3 \mathrm{k}\right)$, 
(ii) $T_{n-1} T_{n+1}-T_{n}^{2}=5(-1)^{n+1}\left(2 Q_{1}-2 i-3 k\right)$.

Proof. (i) Using Lemma 1, for $p=q=1$ we get

$$
\begin{aligned}
\hat{r_{1}} \hat{r_{2}} & =2+(1+\sqrt{5}) i+(3-\sqrt{5}) j+(4+\sqrt{5}) k, \\
\hat{r_{2}} \hat{r_{1}} & =2+(1-\sqrt{5}) i+(3+\sqrt{5}) j+(4-\sqrt{5}) k, \\
\hat{r_{1}} \hat{r_{2}}+\hat{r_{2}} \hat{r}_{1} & =4+2 i+6 j+8 k .
\end{aligned}
$$

Hence and by Corollary 2 we have

$$
\begin{aligned}
Q_{n-1} Q_{n+1}-Q_{n}^{2}= & -\frac{1}{5}(-1)^{n-1}[4+2 i+6 j+8 k \\
& +\frac{3-\sqrt{5}}{2}(2+(1+\sqrt{5}) i+(3-\sqrt{5}) j+(4+\sqrt{5}) k) \\
& \left.+\frac{3+\sqrt{5}}{2}(2+(1-\sqrt{5}) i+(3+\sqrt{5}) j+(4-\sqrt{5}) k)\right] \\
= & (-1)^{n}(2+4 j+3 k)=(-1)^{n}\left(2 Q_{1}-2 i-3 k\right) .
\end{aligned}
$$

We omit the proof of (ii).

Proposition 3 Let $\mathrm{n}, \mathrm{p}, \mathrm{q}$ be integers such that $\mathrm{n} \geq 0, \mathrm{p}^{2}+4 \mathrm{q}>0$. Then

$$
H_{n+1} H_{n-1}-H_{n}^{2}=-\alpha \beta(-q)^{n-1}\left(q\left(\hat{r_{1}} \hat{r_{2}}+\hat{r_{2}} \hat{r_{1}}\right)+r_{1}^{2} \hat{r_{1}} \hat{r_{2}}+r_{2}^{2} \hat{r_{2}} \hat{r_{1}}\right) \text {. }
$$

For $p=2$ and $q=1$ we get the Cassini's identity for the split Pell quaternions $S P_{n}$ and the split Pell-Lucas quaternions $S P L_{n}([7])$.

Corollary 4 Let $\mathrm{n} \geq 1$ be an integer. Then

$$
\begin{aligned}
S P_{n+1} S P_{n-1}-S P_{n}^{2} & =(-1)^{n}(2+4 i+2 j+16 k), \\
S P L_{n+1} S P L_{n-1}-S P L_{n}^{2} & =(-1)^{n-1}(4+8 i+4 j+32 k) .
\end{aligned}
$$

Theorem 5 (d'Ocagne's identity) Let $\mathrm{m}, \mathrm{n}, \mathrm{p}, \mathrm{q}$ be integers such that $\mathrm{n} \geq 0$, $\mathrm{p}^{2}+4 \mathrm{q}>0$. Then

$$
H_{n} H_{m+1}-H_{n+1} H_{m}=\frac{(-q)^{m}\left(b-a r_{2}\right)\left(b-a r_{1}\right)}{r_{1}-r_{2}}\left(r_{1}^{n-m} \hat{r}_{1} \hat{r_{2}}-r_{2}^{n-m_{r_{2}} \hat{r_{1}}}\right) \text {, }
$$

where $\hat{r_{1}} \hat{r_{2}}, \hat{r_{2}} \hat{r_{1}}$ are given by (16), (17), resp. 
Proof. By (15) we get

$$
\begin{aligned}
& H_{n} H_{m+1}-H_{n+1} H_{m}=\left(\alpha \hat{r_{1}} r_{1}^{n}-\beta \hat{r_{2}} r_{2}^{n}\right)\left(\alpha \hat{r_{1}} r_{1}^{m+1}-\beta \hat{r_{2}} r_{2}^{m+1}\right) \\
& -\left(\alpha \hat{r_{1}} r_{1}^{n+1}-\beta \hat{r_{2}} r_{2}^{n+1}\right)\left(\alpha \hat{r_{1}} r_{1}^{m}-\beta \hat{r}_{2} r_{2}^{m}\right) \\
& =\alpha \beta\left(r_{1}-r_{2}\right)\left(r_{1}^{n} r_{2}^{m} \hat{r_{1}} \hat{r_{2}}-r_{1}^{m} r_{2}^{n} \hat{r_{2}} \hat{r_{1}}\right) \\
& =\alpha \beta\left(r_{1}-r_{2}\right)\left(r_{1} r_{2}\right)^{m}\left(r_{1}^{n-m} \hat{r_{1}} \hat{r_{2}}-r_{2}^{n-m_{\hat{2}} \hat{r_{1}}}\right) \\
& =\frac{\left(b-a r_{2}\right)\left(b-a r_{1}\right)(-q)^{m}}{r_{1}-r_{2}}\left(r_{1}^{n-m_{\hat{r}} \hat{r_{2}}}-r_{2}^{n-m_{\hat{r}} \hat{r_{1}}}\right) \text {. }
\end{aligned}
$$

In the next theorem we give a summation formula for the split Horadam quaternions.

Theorem 6 Let $\mathrm{n}, \mathrm{p}, \mathrm{q}$ be integers such that $\mathrm{n} \geq 0, \mathrm{p}^{2}+4 \mathrm{q}>0$. Then

$$
\begin{gathered}
\sum_{l=0}^{n} H_{l}=\frac{H_{n+1}+q H_{n}+(a p-a-b)(1+i+j+k)}{p+q-1} \\
-i a-j(a+b)-k(a+b+p b+q a) .
\end{gathered}
$$

Proof. By formula (12) we get

$$
\begin{aligned}
& \sum_{l=0}^{n} H_{l}=\sum_{l=0}^{n} H_{l}+i \sum_{l=0}^{n} H_{l+1}+j \sum_{l=0}^{n} H_{l+2}+k \sum_{l=0}^{n} H_{l+3} \\
= & \frac{1}{p+q-1}\left[W_{n+1}+q W_{n}+a(p-1)-b+i\left(W_{n+2}+q W_{n+1}+a(p-1)-b\right)\right. \\
& \left.+j\left(W_{n+3}+q W_{n+2}+a(p-1)-b\right)+k\left(W_{n+4}+q W_{n+3}+a(p-1)-b\right)\right] \\
& -i W_{0}-j\left(W_{0}+W_{1}\right)-k\left(W_{0}+W_{1}+W_{2}\right) .
\end{aligned}
$$

Hence we obtain

$$
\begin{aligned}
\sum_{l=0}^{n} H_{l}= & \frac{1}{p+q-1}\left[W_{n+1}+i W_{n+2}+j W_{n+3}+k W_{n+4}\right. \\
& +q\left(W_{n}+i W_{n+1}+j W_{n+2}+k W_{n+3}+(a p-a-b)(1+i+j+k)\right] \\
& -i a-j(a+b)-k(a+b+p b+q a) \\
= & \frac{H_{n+1}+q H_{n}+(a p-a-b)(1+i+j+k)}{p+q-1} \\
& -i a-j(a+b)-k(a+b+p b+q a) .
\end{aligned}
$$

For $p=q=1$ and $a=0, b=1$ we get the result for the split Fibonacci quaternions $Q_{n}([1])$. 
Corollary $5 \sum_{l=1}^{n} Q_{l}=Q_{n+2}-Q_{2}$.

Now we will give the generating function of the split Horadam quaternions.

Theorem 7 The generating function of the split Horadam quaternions is

$$
f(x)=\frac{H_{0}+\left(H_{1}-p H_{0}\right) x}{1-p x-q x^{2}} .
$$

Proof. Let $\mathrm{f}(\mathrm{x})=\mathrm{H}_{0}+\mathrm{H}_{1} \mathrm{x}+\mathrm{H}_{2} \mathrm{x}^{2}+\ldots+\mathrm{H}_{n} \mathrm{x}^{\mathrm{n}}+\ldots$. Then

$$
\begin{aligned}
\operatorname{pxf}(x) & =\mathrm{pH}_{0} x+\mathrm{pH}_{1} x^{2}+\mathrm{pH}_{2} x^{3}+\ldots+\mathrm{pH}_{n-1} x^{n}+\ldots \\
q x^{2} f(x) & =\mathrm{qH}_{0} x^{2}+\mathrm{qH}_{1} x^{3}+\mathrm{qH}_{2} x^{4}+\ldots+\mathrm{qH}_{n-2} x^{n}+\ldots
\end{aligned}
$$

Hence, by Proposition 2, we get

$$
\begin{aligned}
& f(x)-p x f(x)-q x^{2} f(x) \\
& =H_{0}+\left(H_{1}-p H_{0}\right) x+\left(H_{2}-p H_{1}-q H_{0}\right) x^{2}+\ldots \\
& =H_{0}+\left(H_{1}-p H_{0}\right) x .
\end{aligned}
$$

Thus

$$
f(x)=\frac{H_{0}+\left(H_{1}-p H_{0}\right) x}{1-p x-q x^{2}} .
$$

Moreover, by (14) we obtain

$$
\begin{aligned}
\mathrm{H}_{0} & =a+i b+j(p b+q a)+k\left(p^{2} b+p q a+q b\right), \\
H_{1}-p H_{0} & =b-p a+i q a+j q b+k\left(p q b+q^{2} a\right) .
\end{aligned}
$$

\section{References}

[1] M. Akyiğit, H. H. Kösal, M. Tosun, Split Fibonacci Quaternions, Adv. Appl. Clifford Algebr., 23 (2013), 535-545.

[2] J. Cockle, On systems of algebra involving more than one imaginary, Philosophical Magazine III, 35(238) (1849), 434-435.

[3] A. F. Horadam, Basic properties of a certain generalized sequence of numbers, Fibonacci Quart., 3.3 (1965), 161-176. 
[4] A. F. Horadam, Complex Fibonacci Numbers and Fibonacci Quaternions, Amer. Math. Monthly, 70 (1963), 289-291.

[5] A. A. Pogoruy, R. M. R. Rodrigues-Dagnino, Some algebraic and Analytical Properties of Coquaternion Algebra, Adv. Appl. Clifford Algebr., 20 (2010), 79-84.

[6] E. Polatli, C. Kizilates, S. Kesim, On Split k-Fibonacci and k-Lucas Quaternions, Adv. Appl. Clifford Algebr., 26 (2016), 353-362.

[7] Ü. Tokeşer, Z. Ünal, G. Bilgici, Split Pell and Pell-Lucas Quaternions, Adv. Appl. Clifford Algebr., 27 (2017), 1881-1893.

Received: August 21, 2019 